\title{
Votre eau potable est-elle salubre? Tout dépend de l'endroit où vous vivez!
}

$\mathrm{L}$ a tragédie de l'eau contaminée survenue à Walkerton (Ontario) en 2000 , qui a causé la mort de 7 personnes et en a rendu malades au moins 2300 autres, a ébranlé la confiance des Canadiens en la sécurité de notre approvisionnement public en eau. Après 8 ans et des millions de dollars dépensés en réglementation, les problèmes systémiques persistent néanmoins. L'Ontario a fait plusieurs améliorations importantes et la plupart des provinces ont resserré un peu leur surveillance, mais il reste une lacune structurelle inhérente. Pour assurer uniformément la salubrité de l'eau, il faut des systèmes de traitement bien équipés et un personnel bien formé, mais nous délestons cette responsabilité sur les administrations locales. Les grandes municipalités se débrouillent assez bien, mais beaucoup de communautés plus petites et éloignées ne peuvent pas relever tous les défis techniques et administratifs. Par exemple, les avis de faire bouillir l'eau - moyen de dernier recours - sont trop fréquents. Nous permettons l'existence d'un système à deux vitesses d'approvisionnement en eau, réparti à peu près suivant le clivage urbain-rural.

Même si l'on s'oppose souvent aux améliorations nécessaires en affirmant qu'elles coûtent trop cher, les Canadiens consomment le plus d'eau domestique par habitant au monde et notre coût est parmi les plus bas au monde. Le manque de volonté politique de relever le défi est issu de notre indifférence. Par exemple, l'Ontario a mis de côté un rapport produit en 2005 par un groupe d'experts qui recommandait de regrouper les réseaux de distribution de l'eau afin de produire des économies d'échelle, de procéder à une analyse complète des coûts et de fixer le prix de l'eau de façon à garantir la viabilité et la salubrité de l'approvisionnement ${ }^{1}$. L'Alberta a fait quelques progrès en régionalisant des aqueducs de distribution d'eau traitée, ce qui a amélioré la salubrité de l'eau dans les petites localités. Ces interventions se butent toujours à des difficultés techniques (p. ex., beaucoup de petits réseaux éloignés sont difficiles à atteindre) et à l'opposition politique (p. ex., certaines localités ne veulent pas céder le contrôle de leur service public de distribution de l'eau).

L'Australie a connu des difficultés semblables et les a surmontées. En Australie de l'Ouest et en Australie du Sud, des sociétés d'État sont responsables de l'eau potable dans toutes les localités. En I993, l’État de Victoria a regroupé quelque 120 petites organisations en I5 entreprises viables de distribution de l'eau. L'industrie de l'eau en Australie a maintenant atteint un niveau de cohérence et de maturité qui lui permet d'influencer les politiques publiques et d'établir son propre programme de recherche.

Le Canada pourrait faire de même. Un leadership éclairé et des incitatifs appropriés pourraient permettre à des régies régionales de l'eau de plus grande envergure de réaliser des économies d'échelle et de produire l'expertise technique et administrative nécessaire pour garantir de l'eau salubre à tous les Canadiens. Dans les petites communautés éloignées, y compris beaucoup de réserves des Premières nations, il faut poursuivre le regroupement régional de l'expertise opérationnelle et administrative en la combinant à une technologie moderne de télésurveillance. Peu importe le lieu, il est essentiel de mesurer toute l'eau distribuée et de facturer les coûts réels de garantir la salubrité de l'eau. Les distributeurs d'eau doivent en outre investir pour rémunérer et former les membres du personnel et les reconnaître comme des professionnels essentiels de la santé publique.

En ne tenant pas compte des lacunes du système et en négligeant de franchir les étapes logiques pour améliorer les réseaux municipaux de distribution de l'eau, nous favorisons l'émergence d'un clivage entre les citoyens qui dépendent de l'approvisionnement municipal et ceux qui ont les ressources nécessaires pour s'acheter un appareil de traitement de l'eau à domicile ou pour boire de l'eau embouteillée qui coûte Iooo fois plus cher que ce que nous payons habituellement pour une bonne eau au robinet. Au Canada, la consommation d'eau embouteillée par habitant a augmenté de $40 \%$ entre 1999 et $2004^{2}$. Curieusement, ces produits gagnent en popularité alors même que la plupart des Canadiens semblent indifférents à la nécessité d'investir dans des réseaux communautaires de distribution d'eau de grande qualité. Si la tendance se maintient, le Canada pourrait se retrouver dans la même situation que les régions les plus pauvres du monde, où seuls les riches ont accès à une eau potable salubre de grande qualité.

Plus que la majorité des citoyens, les professionnels de la santé savent que la distribution d'eau salubre, l'immunisation et le développement des antibiotiques sont les interventions qui ont le plus contribué à améliorer la santé des populations au cours du dernier siècle. Vous, nos lecteurs, êtes en mesure d'influencer l'évolution des réseaux communautaires de distribution d'eau potable au Canada.

Vous pouvez commencer par évaluer la compétence de votre service local de distribution d'eau potable. Posez des questions au sujet des titres et qualités de son personnel et des mesures prises pour garantir la salubrité de l'eau. L'entreprise publique mesure-telle l'eau distribuée en installant des compteurs et investit-elle tous ses revenus dans l'amélioration de son service? Contestez les politiciens locaux qui utilisent le service d'eau comme une vache à lait pour financer des projets qui n'ont rien à voir, qui s'opposent aux investissements sensés dans les réseaux d'eau potable en affirmant qu'ils coûtent trop cher, ou qui s'opposent aux options sensées de régionalisation. Les réseaux communautaires de distribution d'eau potable salubre constituent un de nos meilleurs investissements dans la santé publique. Nous n'avons certainement pas besoin d'une autre catastrophe comme celle de Walkerton pour nous aider à comprendre la véritable valeur de notre approvisionnement communautaire en eau potable.

\section{Steve E. Hrudey DSc(ing.) PhD}

Professeur émérite, École de santé publique

Université de l'Alberta

Edmonton, Alta.

Avec l'équipe de rédaction de l'éditorial (Paul C. Hébert MD MHSc, Matthew B. Stanbrook MD PhD, Barbara Sibbald BJ,

Ken Flegel MDCM MSc, Noni MacDonald MD MSc et Amir Attaran LLB DPhil)

Intérêts concurrents : Aucuns déclarés.

\section{RÉFÉRENCES}

I. À toute épreuve : l'importance d'améliorer le secteur ontarien de l'eau. Rapport du Groupe d'experts- Stratégie hydraulique; Swain H, Lazar F, Pine J. Toronto : Imprimeur de la Reine pour l'Ontario; 2005. Disponible : www.pir.gov.on.ca/english laboutpir/publications/Watertight-panel_report_FR.pdf (consulté le 4 mars 2008).

2. Gleick PH, Wolff GH, Cooley H. Table I3 : Per Capita Bottled Water Consumption, by Country, 1999 to 2004. Dans : The world's water, the biennial report on freshwater resources : 2006-2007. Washington : Island Press; 2006. p. 284-286. Disponible à : www.worldwater.org/data20062007/Tableı3.pdf (consulté le 5 mars 2008). 